\title{
Programa de visita domiciliaria del sistema de salud costarricense: lineamientos para su mejora $^{1}$
}

\section{Institución: Universidad de Costa Rica}

\author{
Katherine Solís Cordero ${ }^{2}$ \\ Giancarlo Guevara Francesa ${ }^{3}$
}

Solís, K. y Guevara, G. (2015). Programa de visita domiciliaria del sistema de salud costarricense: lineamientos para su mejora. Rev. Enfermería Actual en Costa Rica, 29, 1-18. DOI:

http://dx.doi.org/10.15517/revenf.v0i29.19671

\section{CÓMO CITAR}

\section{RESUMEN}

Introducción. La visita domiciliaria se constituye en la principal estrategia de atención primaria al acercar los servicios de salud a los domicilios y lugares de trabajo de las personas, lo cual permite conocer de primera mano las necesidades de la población. La visita domiciliaria efectuada por el ATAP representa el primer contacto del individuo, familia y comunidad con el sistema de salud, con importantes beneficios tanto a nivel individual como colectivo. La presente investigación responde a la necesidad de identificar los elementos que el programa de visita domiciliaria requiere mejorar, modificar o sustituir con el fin de maximizar la prestación de este servicio.

Método. Se diseñó un estudio cualitativo, analítico observacional. Se recolectó los datos mediante revisión bibliográfica, entrevista a informantes claves y grupo focal. El análisis se realizó a partir de la teoría fundamentada.

Resultados. Los datos evidenciaron la existencia de elementos a nivel de sistema de salud, programa de visita domiciliaria y figura del ATAP que deben ser revisados, modificados o sustituidos con el fin de que la visita domiciliaria tenga mayores y mejores alcances para la población y el sistema de salud.

Conclusión. El programa de visita domiciliaria representa una fortaleza del sistema de salud costarricense al abordar las inequidades en salud. Sin embargo, es imperativa la toma de decisiones e implementación de acciones que promuevan el mejoramiento y aumento de los alcances que tiene la visita domiciliaria en el abordaje de la población a nivel familiar y comunitario.

Palabras claves: atención-primaria-de-salud, enfermería, salud, salud-pública, visita-domiciliaria

\footnotetext{
${ }^{1}$ Fecha de recepción: 4 de marzo del 2015

Fecha de aceptación: 11 de mayo del 2015

${ }^{2}$ Enfermera, Docente e investigadora. Escuela de Enfermería, Universidad de Costa Rica, Costa Rica. Correo electrónico: katherine.solis22@gmail.com

${ }^{3}$ Enfermero, Supervisor de enfermería. Área de Salud de Pavas. Costa Rica. Correo electrónico: gian4418@hotmail.com
} 




\title{
Program home visit Costa Rica's health system: guidelines for improvement ${ }^{1}$
}

\author{
Institution: University of Costa Rica
}

Katherine Solís Cordero ${ }^{2}$ Giancarlo Guevara Francesa ${ }^{3}$

\section{CITED}

Solís, K. y Guevara, G. (2015). Program home visit Costa Rica's health system: guidelines for improvement. Rev. Enfermería Actual en Costa Rica, 29, 1-18. DOI: http://dx.doi.org/10.15517/revenf.v0i29.19671

\begin{abstract}
Introduction. Home visit is the main strategy of primary care by bringing health services to the homes and workplaces of people, which allows knowing the needs of the population firsthand. Thus, home visit by the ATAP represents the first contact of the individual, family and community with the health system, with significant benefits both individually and collectively. This research responds to the need to identify the elements that the home visiting program needs to improve modify or replace in order to maximize the provision of this service.

Method. It is a qualitative, observational analytic study. Data were collected through documentary research, key informant interviews and focus group. The analysis was performed from the grounded theory.

Results. The main results showed the existence of elements at the level of the health system, home visiting program and the figure of the ATAP that should be reviewed, modified or replaced to the home visit, thus it has bigger and better results for the population and the health system.

Conclusion. The Home Visiting Program is strength of the Costa Rican health system to address health inequities. However, it is imperative to make decisions and implementation of actions that promote the improvement and increased results of the home visit at a family and community level.
\end{abstract}

Keywords: health, home-visit, nursing, primary-health-care, public-health

\footnotetext{
${ }^{1}$ Date of receipt: March 4, 2015

Date of acceptance: May 11, 2015

${ }^{2}$ Nurse. Professor and Researcher of the School of Nursing of the University of Costa Rica. Costa Rica. E mail:

Katherine.solis22@gmail.com

${ }^{3}$ Nurse. Nursing supervisor. Health area of Pavas. Costa Rica. E mail: gian4418@gmail.com
} 


\section{INTRODUCCIÓN}

En Costa Rica, en la década de los 70 se dio los primeros pasos en atención primaria de salud por medio de los programas de Salud Rural y el de Salud Comunitaria Urbano, para cuya ejecución se requirió la formación, por primera vez en el país, del Asistente de Salud Rural y el Asistente de Salud Comunitaria Urbano. De acuerdo con Vargas (2006), la visita a las viviendas se constituyó en la estrategia central de ambos programas, en los que cada encargado debía visitar entre ocho y diez viviendas cada día, de modo que cada vivienda era visitada tres o cuatro veces por año.

Según Villegas et al. (1974), durante la visita se llevaban a cabo diversas actividades como vacunación, control de crecimiento y desarrollo de niños menores de 6 años, control prenatal en el domicilio y promoción del parto en las maternidades, durante las que se identificó signos de alarma para referir a un usuario a la consulta médica, se brindó orientación a mujeres en edad fértil en cuanto a planificación familiar, así como sobre desparasitación periódica, uso de sales de rehidratación oral, promoción de la organización comunitaria y participación de la población en la eliminación de criaderos de vectores de malaria y dengue.

En torno al tema, Vargas (2011) expone que la visita domiciliaria se mantiene como una actividad relevante en la Segunda Reforma del Sistema de Salud de la década de los 90, cuando ambos programas se fusionaron en uno: el Programa de Atención Primaria, con cuya implementación se buscaba desarrollar acciones "por medio de tecnologías prácticas, de bajo costo económico, destinadas a satisfacer necesidades básicas de la salud de los individuos, mediante la participación activa de éstos" (Caja Costarricense del Seguro Social [CCSS], 2003). Considerando lo anterior, la visita domiciliaria ejecutada por el Asistente Técnico de Atención Primaria (ATAP) se ha constituido en un pilar fundamental para aumentar la cobertura y el acceso a los servicios de salud, servicio en el que se intercambia información entre la familia y el técnico en cuanto a las condiciones de la vivienda, las características socioeconómicas de los integrantes de la familia, la condición de salud de cada uno (patologías, control médico, vacunación, entre otras) y el ambiente físico.

En este caso, el ATAP es la figura encargada de realizar la visita domiciliaria del primer nivel de atención en el Sistema de Salud costarricense: si bien su formación es menor al grado técnico, para facilitar la lectura del documento se hará referencia a estos como personal técnico.

En Costa Rica, la visita domiciliaria representa un servicio de salud que garantiza los principios filosóficos de la seguridad social en el país, universalidad, solidaridad, igualdad y equidad (Caja Costarricense de Seguro Social [CCSS], 2013), así como los valores de la Atención Primaria de Salud, dígase derecho a la salud, equidad y solidaridad (Organización Panamericana de la Salud [OPS], 2007), lo cual se aplica cuando todas las personas reciben atención a su salud durante la visita domiciliaria independientemente de su condición de aseguramiento o migratoria.

Al respecto, Cruz y Marthe (2010) destacan que la visita domiciliaria del primer nivel de atención representa una oportunidad de implementar intervenciones para promover acciones preventivas, curativas y de promoción de la salud a los individuos en su contexto familiar por permitir una mayor aproximación del personal 
de salud con la realidad y dinámica de las familias; por consiguiente, es una herramienta óptima para orientar, informar, educar y establecer alianzas con las familias para el empoderamiento y promoción de la autonomía en el autocuidado de la salud tanto individual como colectiva.

Consecuentemente, la visita domiciliaria genera importantes beneficios tanto individuales como sociales: mejora la calidad de vida de las personas con el fin de evitar e internamientos y procesos de rehabilitación que generan grandes costos económicos al sistema de salud, así como empeoramiento de los índices de salud y pérdidas de recurso humano por complicaciones y defunciones asociadas.

No obstante, a pesar de que la población costarricense ha cambiado y presenta diferentes necesidades que se deben abordar, la metodología al implementar el modelo de atención de salud durante la visita domiciliaria se ha mantenido desde sus inicios con el Programa de Salud Rural, así como los aspectos por considerar en la formación del ATAP, a partir de lo que se ha identificado diversas debilidades en el abordaje, lo cual obliga a evaluarlo a partir de los alcances de la intervención del ATAP a nivel domiciliar.

Como profesionales en enfermería, es fundamental conocer el alcance de la visita domiciliaria desarrollada por el ATAP ya que el profesional en enfermería ejerce un papel muy activo desde la formación de este recurso hasta la coordinación, planeamiento, supervisión y evaluación del trabajo realizado por éste y su permanente capacitación.

No obstante, la evaluación, monitoreo y control sobre la labor del ATAP en la visita domiciliaria no ha trascendido del aspecto cuantitativo referente a cuántas visitas diarias realizan, cantidad y edad de las personas atendidas, cantidad de vacunas aplicadas, entre otras, razón por la que se ha llegado a subestimar la importancia de aspectos cualitativos que deben considerarse en cuanto al alcance de la intervención a nivel domiciliar, así lo confirma Centeno (2011), quien afirma que

a pesar que se conoce la importancia del trabajo de estos funcionarios, el sistema de salud, en especial sus jerarquías, no se han centrado en realizar estudios acerca del impacto que tiene la labor de los Asistentes Técnicos de Atención Primaria en la salud de los costarricenses, no se han hecho estudios que reflejen las causas de las bajas coberturas en la visita domiciliar, ni estudios para evidenciar la calidad de atención en la prestación del servicio o bien si las familias usuarias de este servicio mantienen otras perspectivas de la visita domiciliar (p.12).

La presente investigación responde a la necesidad de identificar los elementos que el programa de visita domiciliaria requiere mejorar, modificar o sustituir con el fin de maximizar la prestación de este servicio. Para lograrlo, se parte de la caracterización del Programa de Visita Domiciliaria y de la figura del ATAP, con el fin de que se conozcan para poder comprender mejor los lineamientos que en este documento se proponen para el mejoramiento del programa.

\section{MATERIALES Y MÉTODOS}

Para realizar la investigación, se seleccionó la población adscrita al Área de Salud de Pavas, durante el año 2013, así como al personal técnico que labora en dicho sector. 
Con el fin de lograr los objetivos planteados en el estudio, se trabajó con tres diferentes grupos de población: a. 12 personas de la comunidad que recibieron la visita domiciliaria del ATAP del Área de Salud de Pavas, durante el año 2013, para lo que se consideró la representatividad socioeconómica de los sectores del distrito de Pavas; b. 9 Asistentes técnicos de atención primaria del Área de Salud con un rango de experiencia laboral como personal técnico entre los 4 y 17 años, quienes representaron los sectores de Pavas centro, Rohrmoser, Finca San Juan, Óscar Felipe, Metrópolis, Lomas del Río y Laureles; c. 12 personas con experiencia y conocimiento tanto en atención primaria de salud, como en la historia de la inclusión y formación del ATAP en el sistema de salud costarricense, que laboraron en la CCSS, Ministerio de Salud y/o Universidad de Costa Rica, durante la primera y/o segunda reforma del sector salud de Costa Rica.

La investigación se desarrolló desde un enfoque cualitativo ya que, según Strauss y Corbin (2002), cualquier tipo de investigación que produce hallazgos a los que no se llega por medio de procedimientos estadísticos $\mathrm{u}$ otros medios de cuantificación (...); se trata de un proceso no matemático de interpretación, realizado con el propósito de descubrir conceptos y relaciones en los datos brutos y luego organizarlos en un esquema explicativo teórico. Dado lo anterior, el enfoque cualitativo permitió el análisis del fenómeno en estudio a partir del conocimiento de variables cualitativas, no medibles numéricamente, como la caracterización de la visita domiciliaria y las recomendaciones para su mejoramiento. Además, dadas las características y fines de la presente investigación, corresponde a un estudio de tipo analítico observacional.

Las técnicas de recolección de datos utilizadas fueron la revisión documental, entrevista no estructurada a informantes claves y grupo focal. Para la revisión documental, las fuentes de información incluyeron libros, trabajos finales de graduación, artículos científicos, memorias institucionales, oficios, entre otros documentos referentes principalmente a salud, atención primaria de salud, historia y evolución de la figura del ATAP, visita domiciliaria, reforma del sector salud costarricense, para lo cual se creó una guía de revisión documental a partir de las categorías y subcategorías de la investigación.

Se elaboró dos guías de entrevistas de acuerdo con las características de cada grupo de informantes claves: la primera dirigida a las personas expertas en el tema y la segunda, a representantes de la comunidad donde los ATAP llevan a cabo sus labores. Por último, se construyó una guía de reactivos o preguntas generadoras para el grupo focal que se desarrolló con la participación exclusiva de los ATAP.

Para el procesamiento de la información obtenida por medio de las técnicas cualitativas se empleó el programa de análisis cualitativo de datos Atlas ti. Para el análisis de los datos, se parte de la teoría fundamentada, la cual establece que el análisis no es un proceso estructurado, estático, o rígido, sino de flujo libre y creativo en el que los analistas van de un lado a otro entre tipos de codificación, usando con libertad técnicas analíticas y procedimientos y respondiendo a la tarea analítica que se plantea (Strauss y Corbin, 2002, p. 64).

\section{Consideraciones éticas.}

En la investigación se consideró principios bioéticos (Blázquez, 2004), como el autonomía, ya que las personas participantes del estudio no fueron sometidas a ningún tipo de presión psicológica, por el contrario, su decisión de participar fue voluntaria y tenían el derecho de retirarse o rehusarse a participar en caso de considerarlo necesario; de igual forma, se cumplió con el principio de beneficencia, dado que el principal objetivo de la investigación es promover el bienestar de la población al mejorar la visita domiciliaria; el principio de no maleficencia se respetó, puesto que ninguno de los participantes sufrió algún daño o perjuicio. En cuanto al 
principio de justicia, todas las personas fueron tratadas con igualdad y respeto, mientras que se aseguró la confidencialidad de las personas participantes, a quienes se informó sobre su participación anónima.

\section{RESULTADOS}

A continuación, se presenta los resultados de la investigación en tres apartados: 1. La caracterización del programa de visita domiciliaria, 2. La figura del ATAP, ya que este es el principal recurso humano encargado de realizar la visita domiciliaria del primer nivel de atención del sistema de salud costarricense y 3. Los lineamientos para el mejoramiento del programa de visita domiciliaria, orientados en tres ejes: sistema de salud, Programa de Visita Domiciliaria y figura del ATAP.

\section{Caracterización del Programa de Visita Domiciliaria}

Según el Manual normativo de visita domiciliaria de atención primaria primer nivel de atención de la CCSS (2003), el ATAP debe realizar entre 8 y 10 visitas domiciliarias por día, las cuales para ser consideradas efectivas deben comprender tres acciones como mínimo, entre ellas toma de presión arterial, vacunación, toma de medidas antropométricas, entrega de desparasitantes y suero de rehidratación oral, entrega de referencias y educación en salud.

Este manual expone las etapas de la visita domiciliaria que debe llevar a cabo el ATAP: planeamiento, ejecución y reporte. Durante el planeamiento, el personal prepara las fichas familiares de las viviendas que visitará en las que anota con un lápiz las acciones por realizar y prepara el equipo completo que utilizará según lo planeado.

De acuerdo con una de las personas expertas entrevistadas, esta primera etapa es fundamental para el éxito de la vista domiciliaria: "Cómo abordar la visita domiciliaria depende de la etapa de planeamiento; antes de hacer la visita domiciliaria se debe tener una programación, en la que se hace un análisis y un plan familiar que me permita abordar las necesidades de esa familia" (RM01, 2013).

En la etapa de ejecución se implementa las acciones planeadas y la realización de otras respecto de los hallazgos encontrados durante la visita, las normas establecidas y su perfil laboral. En este momento, se desarrolla las actividades que permiten considerar la visita efectiva o inefectiva.

Durante la visita se efectúa un intercambio de información entre la familia y el técnico, mediante la que se conoce principalmente las condiciones de la vivienda, las características socioeconómicas de los integrantes de la familia, la condición de salud de cada uno (patologías, control médico, vacunación, entre otras) y el ambiente físico. A la vez, brinda la oportunidad de desarrollar actividades de promoción de la salud como la educación en salud y la prevención de la enfermedad por medio de acciones como la vacunación.

Por último, durante el reporte de la visita, el ATAP debe completar adecuadamente los datos de la ficha familiar y la hoja de control individual indicando siempre los hallazgos encontrados, las acciones, recomendaciones y educación brindada, además, debe referir al EBAIS o a la enfermera situaciones encontradas no resueltas debido a su perfil. 
Para la ejecución de la visita domiciliaria se clasifica las familias de acuerdo con las necesidades encontradas, con un enfoque de riesgo por prioridad en I, II o III, según el que cada familia será visitada una, dos o tres veces por año, considerando que las de prioridad I requieren mayor atención y número de visitas anuales.

\section{Figura del asistente técnico de atención primaria}

La figura del ATAP comenzó con la formación del asistente de salud rural por medio de un curso de capacitación que duró, en promedio, cinco meses. El trabajo de este personal era vigilado por un supervisor de campo (figura eliminada en 1989) y una enfermera profesional del centro de salud.

Luego, se capacita al asistente de salud comunitaria urbano, razón por la que en 1984 se creó un solo curso de formación para los técnicos de ambos programas, quienes eran "personas de las mismas comunidades que brindarían servicios mínimos de salud preventivo-asistenciales” (Vargas, Quesada, y Carrillo, 1972, p. 9).

Las figuras del asistente de salud rural y el asistente salud comunitaria urbana pasaron a ser el ATAP, una persona que tras un proceso de formación de seis meses ejecuta labores de atención en salud básica a nivel domiciliario y comunitario, tanto en zonas rurales como urbanas, y desarrolla acciones de baja complejidad en promoción de la salud y prevención de la enfermedad en beneficio de la comunidad que está bajo su responsabilidad. Este recurso humano es supervisado en forma periódica por el profesional en enfermería del equipo de apoyo, a partir de normas y procedimientos establecidos (CCSS, 2003).

En relación con lo anterior, de acuerdo con Garro (1997), la formación de este personal ha estado bajo la responsabilidad de diferentes instituciones; en sus inicios, el Ministerio de Salud fue la entidad encargada de capacitarlos; posteriormente, en los años 90, el Centro de Desarrollo Estratégico e Información en Salud y Seguridad Social (CENDEISSS) asumió el proceso de formación de este personal.

Por último, la Universidad de Costa Rica a través de la Escuela de Enfermería asumió la instrucción de los asistentes técnicos de atención primaria por medio de un curso de formación teórico-práctico de metodología participativa, impartido por docentes de la Escuela de Enfermería, con participación de otras unidades académicas como nutrición, farmacia, microbiología, trabajo social, odontología, así como otras instituciones. La metodología planteada consiste en un curso teórico-práctico con un total de 1085 horas distribuidas en 360 horas teóricas y 725 de práctica; por lo tanto, la persona que finaliza este curso está preparada para incorporarse a las labores del EBAIS. Actualmente, la Universidad de Costa Rica es la principal institución formadora de este personal técnico.

De acuerdo con lo establecido en el Reglamento del ATAP en Salud (ATAPS) del Colegio de Enfermeras y Enfermeros de Costa Rica (2012), el perfil del asistente técnico en atención primaria en salud deberá ajustarse a lo siguiente: 1. Realizar visitas programadas según los escenarios domiciliar, escolar, laboral, comunitario según actividades, normas y lineamientos establecidos; 2. Ejecutar labores asistenciales en el campo, delegadas y supervisadas por el profesional de enfermería como toma de presión arterial y temperatura, medidas antropométricas, observación física y mental básica (piel, cabello y dientes), aplicación de vacunas, registros del control de inmunizaciones, participa en el mantenimiento de la cadena de frío y llenado de registros familiares (ficha familiar y otros instrumentos de registro de información familiar); 3. Colaborar con el profesional de enfermería en el abordaje de eventos sujetos a vigilancia epidemiológica en el campo, en detección oportuna, referencia y seguimiento de casos, 4. Detectar y orientar al usuario y referir al profesional de enfermería, las situaciones relacionadas con el saneamiento básico como abastecimiento de agua potable, eliminación de 
excretas, aguas servidas, manejo de desechos sólidos y control del medioambiente amenazado, entre otras funciones.

Es importante conocer las funciones con las que cumple el ATAP dentro del programa ya que su labor es de vital importancia para el cumplimiento de los objetivos, y consecuentemente, el éxito del programa de visita domiciliaria.

\section{Lineamientos para el mejoramiento del programa de visita domiciliaria}

La visita domiciliaria del sistema de salud costarricense representa una fortaleza del modelo de atención de salud al acercar los servicios a los lugares donde se encuentran las personas y garantizar el derecho a la salud. Sin embargo, requiere de modificaciones fundamentales que consideren las nuevas características de la población actual y las necesidades del sistema.

Lo anterior es respaldado con lo expuesto por uno de los ATAP participante en el grupo focal: "Ya es hora que cambie este programa, casi todos los programas de la Caja han sufrido grandes cambios pero el Programa de Atención Primaria lo seguimos arrastrando desde hace más de 30 años con una estructura rígida que va dejando de ser funcional. Creo que es importante que se involucren diferentes actores para que lleven a cabo la reforma que estamos necesitando" (grupo focal, 2013).

Estas modificaciones que se identificaron como necesarias para mejorar el programa deben implementarse en tres niveles: sistema de salud, como un todo dentro del cual se encuentra inmerso el programa de visita domiciliaria, el programa como tal en sus diferentes componentes y, por último, el ATAP, como principal recurso humano encargado del desarrollo de la visita.

A nivel de sistema de salud, uno de los aspectos fundamentales que limita los resultados de la visita domiciliaria es el nulo o poco trabajo interdisciplinario, ante lo cual una de las ATAP participante del grupo focal señaló:

EBAIS es un equipo básico de atención integral en salud pero si nos vamos a ver lo que hay, no hay un equipo y mucho menos atención integral, cada quien trabaja por separado y medicalizado todo. Uno a veces siente que no hace nada aunque sí lo hace, pero es que uno solo no puede hacerlo, de verdad se necesita el equipo. Hubo un momento en el que trabajamos así la doctora, la comunidad y yo, pero ahora no se hace (grupo focal, 2013).

Por lo tanto, es importante incluir todo el equipo en la atención de las necesidades de la población extramuros, con el fin de conocer las condiciones reales en las que viven las personas y los efectos directos sobre su situación de salud.

Por otra parte, se identificó que el carente trabajo articulado entre los miembros del EBAIS y el equipo de apoyo del área limita el accionar del ATAP en cuanto a la solución de los problemas encontrados durante la visita domiciliaria: "Tenemos que pensar que el ATAP no trabaja solo, él es parte de un sistema, son las políticas, es enfermería, no él, el ATAP no trabaja solo, él es mandado" (MS01, 2013). 
De esta forma, el apoyo que el ATAP recibe por parte de todos los miembros del EBAIS y del equipo de apoyo es indispensable para cumplir con una atención integral en salud; por ende, se debe dar mayor validez al criterio del técnico al momento de referir situaciones especiales detectadas, sustentadas en que el ATAP es quien posee un conocimiento diferenciado y global de la situación de su comunidad.

Además, es indispensable que por medio de políticas o normas se le facilite al personal técnico, como parte del EBAIS, el proceso de referencia de situaciones especiales encontradas que deben ser atendidas por profesionales del equipo de salud o el equipo de apoyo.

Por otra parte, a nivel de sistema de salud, la intersectorialidad representa una de sus debilidades, lo cual se observa en la siguiente cita: “¿Cuál es la relación o conexión que tiene el sistema con otros sectores o instituciones? Esto por sí solo anula en muchos casos la intervención del ATAP. No solo no se logran resultados por el ATAP solo, sino que el sistema le limita al ATAP su trabajo. Repito el ATAP no puede trabajar solo y la intersectorialidad es fundamental" (RM01, 2013). Otra de las expertas expresó: "Hay una falla estructural desde arriba, que está afectando la visita domiciliaria, no es el ATAP ni la visita en sí, es un sistema que no ha hecho la transición de la curación a la promoción y la prevención" (MS01, 2013).

Otra debilidad del sistema que tiene implicaciones negativas sobre el programa de visita domiciliaria se refiere a implementar los compromisos de gestión ya que evaluar el quehacer del ATAP se ha limitado principalmente al aspecto cuantitativo, con el fin de lograr el cumplimiento de las coberturas establecidas en los compromisos de gestión, hecho sobre el que una de las personas expertas manifestó: "Los compromisos de gestión han limitado los beneficios que pueden obtener las personas de la visita domiciliaria ya que no buscan la calidad, sino la cantidad" (RM01,2013).

Otro aspecto relevante que tiene consecuencias en la visita domiciliaria es la ausencia de sistemas de información digitalizados en todas las áreas de salud del país, lo cual ha provocado un uso limitado de la información recolectada por los ATAP, situación que es respaldada por uno de los expertos entrevistados: "Es increíble la cantidad de datos que nosotros tenemos olvidados en las fichas familiares, a nivel nacional existe toda la información de la población visitada pero al no tener bases de datos, la información se pierde" (PO01, 2013).

De esta forma, se constata la necesidad de contar con un sistema de información en el que se genere bases de datos donde se encuentre la información recolectada durante la visita domiciliaria, para analizar datos a nivel local y nacional de las necesidades que identifica el ATAP, según área de salud, con el fin de que otras instituciones y sectores tengan acceso a estos datos.

En cuanto al nivel del programa de visita domiciliaria, una vez más el enfoque cuantitativo desde el que se ha evaluado la visita domiciliaria, centrado en la cantidad de visitas realizadas por cada ATAP, se destaca como una de las principales debilidades del programa. Lo anterior es respaldado con las apreciaciones de los ATAP participantes:

Además, revisar la cantidad porque esto parece una maquila donde lo que se pide es cantidad pero, ¿quién revisa la calidad del producto que sale? La Caja no nos puede seguir calificando así por números, somos un gran recurso que a lo mejor no se está usando como debería. La salud no es estadística, hay mucho más detrás de esos números que no se ve (grupo focal, 2013). 


\section{Revista Electrónica Enfermeria Actual en costa Rica}

Por otra parte, los ATAP consideran que para un mayor impacto de la visita domiciliaria es necesario revisar la clasificación de las familias según el riesgo, por tanto,

hay que eliminar cosas e impulsar cosas nuevas, hay que eliminar la visita de prioridad I tres veces al año, muchas veces eso no es necesario, habrá ocasiones en las que puede ser necesaria una vigilancia de hasta 10 veces al año pero hay cosas que no tienen sentido (grupo focal, 2013).

Además de mantener la clasificación de riesgo de las familias, es fundamental revisar el instrumento utilizado para llevar a cabo dicha clasificación, desde el enfoque de determinantes sociales de la salud, considerando la existencia de determinantes estructurales e intermedios que imposibilitan que el ATAP genere un mayor impacto asociado a la cantidad de visitas realizadas.

Aunado a la anterior, una medida para mejorar el servicio se refiere a los escenarios donde se realizan las visitas, acerca de lo que una participante del grupo focal manifestó:

Yo sé que la visita tiene que cambiar, tiene que evolucionar y ser diferente porque todo cambia, si ya no puedo ir a la casa porque la persona no está ahí puedo ir al trabajo, hay que implementar la visita laboral porque el ausentismo va a ser cada vez mayor. Se debe innovar mucho, la idea de llevar la visita al espacio laboral permite la proyección a los jefes y jefas de familia lo que sería muy enriquecedor porque lo llevan a sus casas (grupo focal, 2013).

Otra de las categorías de análisis que surgió de los resultados obtenidos fue el papel del profesional de enfermería en el Programa de Visita Domiciliaria: en su mayoría, las personas expertas señalaron que las principales debilidades se asocian fundamentalmente a una escasa participación del profesional de enfermería en el desarrollo de la visita domiciliaria y su rol como supervisor, lo cual está asociado a la problemática que enfrenta el gremio dado que se ha reducido la contratación y número de plazas de profesionales de enfermería en el primer nivel de atención, así como su ubicación como parte del equipo de apoyo del Área de Salud y su consecuente recargo de funciones.

Enfermería realizó una labor protagónica junto con el ATAP en la comunidad, no obstante, se ha observado un declive en esta participación del profesional de enfermería como líder del Programa de Visita Domiciliaria, desde el traspaso del mismo del Ministerio de Salud a la CCSS, por ello un entrevistado afirma que

la visita domiciliar hay que recuperarla, y creo que enfermería es la culpable: enfermería perdió la visión de la visita, se centró en compromisos de gestión y papeleo, por lo que se desvinculó del ATAP. Claro, es necesario que tengamos más colegas en el primer nivel de atención. ¿Cómo es posible que hayan colegas que tengan a cargo 15 o más ATAPS? Ha habido un aumento en la población y se ha mantenido la misma cantidad de recurso humano, por ello ha bajado la calidad y la cantidad de lo que hacían o que no realizan (AA01, 2013).

Por otra parte, durante la recolección de datos, surgieron varias recomendaciones por parte del grupo de personas expertas y el equipo investigador, orientadas al fortalecimiento de la formación del personal técnico y relacionadas con el aumento en el tiempo de formación y la inclusión de temas en el programa de estudio que le proporcionen mayores conocimientos y competencias para enfrentar distintas situaciones durante la visita domiciliaria. 
Con respecto a la inclusión de nuevas temáticas en el plan de estudios del ATAP, las personas expertas entrevistadas enfatizan el abordaje de temas relacionados principalmente con el enfoque de determinantes sociales de la salud y el trabajo con la comunidad. Aunado a ello, los ATAP deben ser formados desde los valores, principios y elementos de un sistema de salud basado en atención primaria de salud.

Además, es fundamental ampliar el tiempo de formación para maximizar el desarrollo de las competencias de este recurso humano, razón por la que las personas expertas sugieren un estudio de la propuesta de formación del ATAP con el fin de que el proceso pueda ampliarse de 9 a 12 meses.

En cuanto al perfil del ATAP, una de las principales críticas a las funciones con las que deben cumplir, dicho recurso humano, se refiere a la presencia cada vez menor en la comunidad y el desarrollo de actividades orientadas al mejoramiento de la calidad de vida colectiva, lo cual queda plasmado en la siguiente opinión:

El ATAP de antes trabajaba con y para la comunidad, ellos conocían su comunidad, había una comunicación directa con la comunidad y había participación comunitaria. Actualmente, la participación comunitaria está totalmente aislada del sistema, y eso no puede ser porque nuestra razón de ser es la comunidad. Y quien conoce la comunidad, quien puede buscar estrategias de intervención y trabajo diferentes es la propia comunidad, pero hemos anulado completamente la participación de la comunidad, se ha llegado a un irrespeto absoluto del trabajo de la comunidad (RM01, 2013).

\section{DISCUSIÓN}

De acuerdo con Villegas (1978), desde la década de los 70, el sistema de salud costarricense invierte en programas que pretendían la extensión de la cobertura y el acceso a los servicios de salud como el Programa de Salud Rural y Salud Urbana Comunitaria. Para la implementación de ambos, fue necesario capacitar a personal que ejecutara las labores propias de este modelo de atención de la salud, principalmente el desarrollo de la visita domiciliaria en el primer nivel de atención, entendida como

una modalidad de los programas de apoyo a la familia, en donde ésta recibe servicios en su propio hogar, a través de encuentros periódicos realizados por un agente visitador previamente definido, permitiendo entregar una amplia gama de servicios, como información, guía y apoyo emocional, entre otros (Kotliarenco y otros, 2010, p.186).

La visita domiciliaria se constituye en la principal estrategia de atención primaria que permite al ATAP observar, describir y detectar los eventos o situaciones -presentes en la comunidad- relacionadas con salud; por lo tanto, representa el instrumento ideal para conocer el medio en el que viven la persona y su familia, el cual influye en la condición de salud de quienes habitan en la vivienda. Lo anterior brinda la oportunidad de llevar a cabo acciones que permitan a las personas apropiarse de nuevos conocimientos para analizar su situación de salud y desarrollar conductas y prácticas que les permita mejorar su proceso salud-enfermedad.

Con base en lo anterior, Sakata (2007), citado por De Araújo y Ribeiro (2011), señala que la visita domiciliaria representa un modo diferente de cuidar la salud, por medio de la que se logra una mayor proximidad con las familias para el desenvolvimiento de intervenciones y acciones de promoción y prevención, por ello es que el acercamiento de los servicios de salud a los domicilios de las personas y los lugares de trabajo permite 
conocer de primera mano las necesidades de la población, en lo que el ATAP representa el primer contacto del individuo, familia y comunidad con el sistema de salud.

Respecto de la visita domiciliar, Centeno (2011) expone que es un espacio creado por el sistema de salud del país, que le brinda la oportunidad a las familias de todas las clases sociales de tener un acercamiento al sistema al que, a su vez, facilita la captación de las personas en programas de salud establecidos como control prenatal, crecimiento y desarrollo, captación de personas con hipertensión arterial, personas con descontrol metabólico (Diabetes mellitus), adultos mayores en estado de abandono, violencia intrafamiliar, esquemas de vacunación incompletos, entre otros.

Sin embargo, cabe destacar que, según Ferreira y de Campos (2001), no toda visita al domicilio del usuario puede ser considerada una visita domiciliaria, ya que para serlo debe comprender un conjunto de acciones sistematizadas que se inician antes y continúan después de la visita en el domicilio de las personas usuarias, además de que precisa de un recurso humano con una capacitación específica. A lo anterior, hay que agregar lo mencionado por Ferreira y de Campos (2001), Cunha y Gama, (2012), Velasco y Durán (2003), quienes coinciden en que la visita domiciliaria en el contexto de la atención primaria en salud debe sistematizarse en tres etapas, planeamiento, ejecución y reporte, momentos que se desarrollan a lo largo del Programa de Visita Domiciliaria (como se presentó en los resultados, el ATAP debe cumplir con tareas específicas en cada una de estas etapas con el fin de que se alcancen los objetivos del programa).

Durante la etapa de ejecución de la visita, el ATAP puede encontrar situaciones que desde su formación básica requieren de un acompañamiento multi e interdisciplinario para dar solución, así el trabajo articulado con el resto de profesionales del equipo es fundamental para que los alcances de su labor sean los esperados por la población y el sistema de salud, lo cual es respaldado por la OPS (2007) ya que

los equipos multidisciplinarios son fundamentales para el enfoque de la atención primaria de salud, lo que no solo requiere de una combinación correcta de profesionales, sino también de una definición adecuada de funciones y responsabilidades, de una distribución geográfica equitativa, y de una capacitación que maximice su contribución a los resultados de salud y a la satisfacción de los trabajadores de salud y de los usuarios" (p. 14-15).

Paralelo al trabajo interdisciplinario, es fundamental la atención de la salud desde el principio de la intersectorialidad expuesto por la estrategia de atención primaria de salud, la cual afirma que un sistema de salud debe facilitar las aportaciones coordinadas e integradas de todos los sectores, incluidos los sectores públicos y privados en torno a los factores determinantes de la salud, con el objetivo de alcanzar el grado máximo de salud que sea posible (OPS, 2007). Todo lo mencionado se fundamenta en la evidencia respecto de que, en general, la carga atribuible que tienen los servicios de salud de atención directa a las personas (servicios médicos) no sobrepasa el 20\%, mientras que aproximadamente el 80\% restante es atribuible a la modificación de factores que están fuera de estos servicios, es decir, por la modificación en factores ambientales, socioeconómicos y culturales (Ministerio de Salud de Costa Rica, 2011). 
De acuerdo con la Comisión de Determinantes Sociales de la Salud de la OMS (2008), este conjunto de factores sociales, políticos, económicos, ambientales y culturales que ejercen gran influencia en el estado de salud se resume en la expresión "determinantes sociales", los cuales constituyen las "las causas de las causas", es decir, la esencia de la jerarquía social del mundo y de cada país, y las condiciones sociales que son producto de dicha jerarquía y que determinan la situación en la que las personas crecen, viven, trabajan y envejecen.

Con base en este enfoque de determinantes sociales de la salud es que se debe revisar, actualizar y modificar el instrumento de clasificación del riesgo utilizado por el ATAP para determinar la prioridad de las familias, debido a que en el instrumento actual se considera determinantes estructurales e intermedios que no pueden ser modificados por el accionar del ATAP. Por medio de dicha revisión, el recurso humano puede brindar mayor criterio en la ejecución de las visitas domiciliarias que realiza con base en el conocimiento y experiencia que posee sobre su comunidad y las personas usuarias adscritas al programa, para definir las situaciones que ameritan un mayor uso del tiempo en el abordaje de situaciones y, por ende, la posible reducción del número total de visitas del día reportadas.

La extensión del accionar del ATAP a otros escenarios fuera del hogar responde a la estrategia de atención primaria de salud que pretende acercar los servicios donde las personas viven, estudian y trabajan (OPS, 2007); por consiguiente, con respecto a la visita a nivel laboral, el Reglamento del Asistente Técnico de Atención Primaria de Salud (ATAPS) establece que el personal técnico debe cumplir con tareas específicas durante la visita en el escenario laboral (Colegio de Enfermeras y Enfermeros de Costa Rica, 2012).

La inclusión de las mujeres en el ámbito laboral, así como largas jornadas de trabajo, y la ausencia de las familias en sus domicilios durante los horarios de visita de los ATAP, motiva el realizar la visita en el escenario laboral con el fin de que las personas que se encuentran en estos espacios puedan recibir los beneficios de este servicio.

De acuerdo con lo estipulado por el Reglamento del Asistente Técnico de Atención Primaria en Salud (ATAPS), el ATAP es un recurso humano que forma parte del equipo de enfermería al ser formado, capacitado y supervisado por los profesionales en enfermería, por lo que la gestión del programa de visita domiciliaria hecha por el profesional de enfermería representa uno de los ejes fundamentales para el desarrollo de una intervención exitosa por parte del ATAP, ya que enfermería es responsable de evaluar, principalmente la calidad de la visita domiciliaria, detectar debilidades y establecer estrategias para su mejora; sin embargo, se aprecia una mayor afinidad al cumplimiento de los compromisos de gestión, asociado también a un recargo de actividades del profesional de enfermería que labora en el primer nivel de atención y también a una formación deficiente sobre atención primaria de salud desde la academia.

Por otra parte, según lo exponen Méndez y Parada, (2001), a pesar de que el 80\% del total de los asistentes técnicos de atención primaria creen que los conocimientos adquiridos en su formación les han permitido enfrentar las demandas de trabajo, dado que un porcentaje alto (63.5\%) -para lo que debería ser este indicador en su estado ideal- considera que existe una marcada diferencia entre lo que aprendió en su formación y el conocimiento que debe aplicar en el desempeño de sus tareas. Lo anterior debe justificar una redefinición de los perfiles ocupacional y educacional de estos funcionarios. Lo agregado por los autores fundamenta la importancia de que el ATAP posea un perfil ocupacional en el que se delimite con claridad sus funciones y tareas dentro del Programa de 
Visita Domiciliaria con el fin de que dedique su tiempo y conocimientos a cumplir con éstas, sin saturarse con actividades fuera de su perfil y que podrían limitar su accionar en el espacio comunitario.

La ausencia de este perfil definido ha ocasionado que el ATAP no esté cumpliendo con una de sus principales funciones, el trabajo en la comunidad, y a la vez se incumpla elemento de participación activa de un sistema de salud basado en Atención Primaria de Salud el cual implica "actividades que facultan a los individuos para que manejen mejor su propia salud y que estimulen la capacidad de las comunidades de convertirse en socios activos en la determinación de prioridades, la gestión, la evaluación y la regulación del sector de la salud" (OPS, 2007, p. 14).

\section{CONCLUSIONES}

El Programa de Visita Domiciliaria del primer nivel de atención se constituye en una fortaleza del sistema de salud costarricense al abordar las inequidades en salud, dado que brinda atención universal y garantiza el derecho a la salud de la población. Sin embargo, debido a las modificaciones sociales, culturales, ambientales y epidemiológicas de la sociedad costarricense en las últimas décadas es imperativa la toma de decisiones e implementación de acciones con las que se mejore y aumente los alcances de la visita domiciliaria en el abordaje de la población a nivel familiar y comunitario.

Esta visita debe seguir siendo realizada por el ATAP: la figura del ATAP dentro del sistema de salud costarricense no debe eliminarse o sustituirse por otro profesional de salud, puesto que según los requerimientos del sistema y los aspectos fundamentales de la estrategia de atención primaria, la figura del ATAP representa el primer contacto entre los individuos, familia y comunidad, dado que es quien brinda asistencia sanitaria esencial a un costo que la comunidad y el país puedan soportar.

La figura del asistente técnico de atención primaria que surgió durante la década de los 70 debe mantenerse como pieza fundamental del sistema de salud costarricense; sin embargo, es indispensable revisar su perfil ocupacional con el fin de adaptar sus tareas y funciones a las características sociales, demográficas y epidemiológicas de la población costarricense.

Como producto de esta investigación se identificó importantes debilidades que requieren una revisión exhaustiva mediante la que se implemente acciones que promuevan el mejoramiento del desarrollo de la visita domiciliaria realizada por el ATAP y el consecuente impacto positivo de los alcances de esta en la salud de la población y las necesidades del sistema de salud.

\section{REFERENCIAS BIBLIOGRÁFICAS}

AA01. (2013). Entrevista a informante clave. (E. investigador, Entrevistador)

Blázquez, N. (2004). La bioética y los hijos del futuro. Madrid: Editorial Visión NET.

Caja Costarricense del Seguro Social. (2003). Manual Normativo de Visita Domiciliar de Atención Primaria Primer Nivel de Atención. San José: Caja Costarricense del Seguro Social. 
Caja Costarricense de Seguro Social . (2013). Memoria Institucional. San José: CCSS.

Centeno, M. (2011). Calidad del trabajo de los asistentes técnicos de atención primaria en la visita domiciliar, desde la efectividad, la aplicación de los procedimientos y la satisfacción del usuario, en el Área de Salud Florencia. San José: Instituo Centroamericano de Administración Pública.

Colegio de Enfermeras y Enfermeros de Costa Rica. (2012). Reglamento del Asistente Técnico de Atención Primaria (ATAPS). San José.

Cruz, M. y Marthe, M. (2010). A visita domiciliária na estratégia de saúde da família: conhecendo as percepções das famílias. Revista Saúde e Sociedade, 605-613.

Cunha, C. y Gama, M. (2012). A VISITA DOMICILIAR NO ÂMBITO DA ATENÇÃO PRIMÁRIA EM SAÚDE. Obtenido de Universidade Federal Fluminense:

http://www.uff.br/tcs2/images/stories/Arquivos/textos_gerais/A_VISITA_DOMICILIAR_NO_MBITO_DA_ATEN O PRIMRIA EM SADE.pdf

De Araújo, A. Y Ribeiro, B. (2011). A visita domiciliaria com ênfase na educação em saúde. Revista Electrónica Saúde:

Pesquisa e Reflexões, 1-16.

Ferreira, R. y de Campos, A. (2001). A visita domiciliária no contexto da saúde da família. En U. d. Paulo, Manual de Enfermagem (págs. 43-46). Sao Paulo: Universidade de Sao Paulo.

Garro, M. (1997). Programa de capacitación dirigido a asistentes técnicos de atención primaria para el manejo del individuo con trastorno mental en la comunidad, Guápiles. Costa Rica: Universidad de Costa Rica.

Grupo Focal. (2013). Alcances de la visita domiciliaria realizada por el asistente técnico de atención primaria. (E. investigador, Entrevistador)

Kotliarenco, M. y otros. (2010). Características, efectividad y desafíos de la visita domiciliaria en programas de intervención temprana. Revista de Salud Pública, 184-196.

Méndez, E. y Parada, N. (2001). Informe de Investigación sobre Necesidades de Capacitación en los Asistentes Técnicos de Atención Primaria (ATAPS). Revista de Ciencias Administrativas y Financieras de la Seguridad Social, 53-69.

Ministerio de Salud de Costa Rica. (2011). Modelo Conceptual y Estratégico de la Rectoría de la Producción Social de la Salud. San José: Ministerio de Salud de Costa Rica.

MS01. (2013). Entrevista a informante clave. (E. investigador, Entrevistador)

Organización Mundial de la Salud. (2008). Subsanar las desigualdades en una generación. Alcanzar la equidad sanitaria actuando sobre los determinantes sociales de la salud. Buenos Aires: Ediciones Journal S.A.

Organización Panamericana de la Salud. (2007). La Renovación de la Atención Primaria de Salud en las Américas. Documento de Posición de la Organización Panamericana de la Salud/Organización Mundial de la Salud (OPS/OMS). Washington, D.C.: Organización Panamericana de la Salud.

PO01. (2013). Entrevista a informante clave. (E. investigador, Entrevistador) 
RM01. (2013). Entrevista a informante clave. (E. investigador, Entrevistador)

RS01. (2013). Entrevista a informante clave. (E. investigador, Entrevistador)

Strauss, A. y Corbin, J. (2002). Bases de la investigación cualitativa. Técnicas y procedimientos para desarrollar la teoría fundamentada. Bogotá: CONTUS-Editorial Universidad de Antioquia.

Vargas, W. (2006). Atención Primaria de Salud en Acción : su contexto histórico, naturaleza y organización en Costa Rica. San José: Editorial Nacional de Salud y Seguridad Social (EDNASSS).

Vargas, W. (2011). Atención primaria de salud en Costa Rica: contexto histórico, naturaleza y organización. San José: EDNASSS-CCCSS.

Vargas, W., Quesada, E. y Carrillo, G. (1972). Manual de Procedimientos para el Asistente de Salud Rural. Costa Rica: Ministerio de Salubridad Pública.

Velasco, A. y Durán, M. (2003). La visita domiciliaria como programa para el abordaje de la familia. Obtenido de http://www.saluddealtura.com/fileadmin/PDF/CAPACITACION/4_DOCUMENTO_2_visita_domiciliaria.pdf

Villegas, H. (1978). Extensión de la cobertura de salud en Costa Rica. Hospitales de Costa Rica, 28-33.

Villegas, H. (2006). Atención Primaria en Salud: Escenarios, renovación, desafios. Obtenido de CENDEISSS: http://www.cendeisss.sa.cr/seguridadsocial/index_archivos/HugoVillegas.pdf

Villegas, H. et al. (1974). Programa de Salud para Comubidades Rurales. San José: Ministerio de Salud. 